\title{
INFRASTRUCTURE The impact of acute stroke service centralisation: a time series evaluation
}

\author{
Authors: Mat Elameer, ${ }^{A}$ Chris Price, ${ }^{B}$ Darren Flynn ${ }^{C}$ and Helen Rodgers ${ }^{D}$
}

\begin{abstract}
We evaluated the impact of the centralisation of three acute stroke units into a single hyperacute stroke unit within a large urban and rural NHS trust in North East England in June 2015. We performed retrospective interrupted time series analyses of 4,305 stroke patients admitted between 1 April 2013 and 31 December 2017 utilising data recorded for the Sentinel Stroke National Audit Programme. Centralisation was associated with reductions in total length of inpatient stay ( -4.9 days [95\% CI: -8.1 to -1.7$]$ ). Time from admission to thrombolysis shortened by 26.0 minutes (95\% CI: -40.0 to -12.1), and time from admission to brain imaging for thrombolysed patients improved by 16.2 minutes ( $95 \%$ CI: -22.0 to -10.4). Time from stroke onset to hospital admission, mortality and dependency (as measured by median modified Rankin Scale) were unaffected by centralisation. This study provides further evidence to support the centralisation of acute stroke services in England.
\end{abstract}

\section{Introduction}

Randomised controlled trials and subsequent meta-analyses have clearly shown that stroke units reduce death and dependency. ${ }^{1}$ Although stroke units are the cornerstone of evidence-based stroke care, there is ongoing debate about whether acute stroke services should be provided locally with greater remote support or centrally with greater physical concentration of resources. ${ }^{2-6}$ Centralisation offers faster access to direct specialist care, neuroimaging and thrombolysis following arrival at hospital, but potentially at the expense of prolonged travel times. ${ }^{7}$ Achieving this balance is particularly important for patients who may be eligible to receive thrombolysis because the time from stroke onset to receiving this treatment is a key determinant of outcome. ${ }^{8}$

The National Health Service Five Year Forward View and proposed development of urgent and emergency care centres ${ }^{9}$ is driving the review of acute stroke services across England.

Authors: Aacademic clinical fellow, Stroke Research Group, Newcastle University, Newcastle-upon-Tyne, UK; ${ }^{B}$ Clinical reader, Stroke Research Group, Newcastle University, Newcastle-uponTyne, UK; C senior research associate, Institute of Health and Society, Newcastle University, Newcastle-upon-Tyne, UK; D professor of stroke care, Stroke Research Group, Newcastle University, Newcastle-upon-Tyne, UK

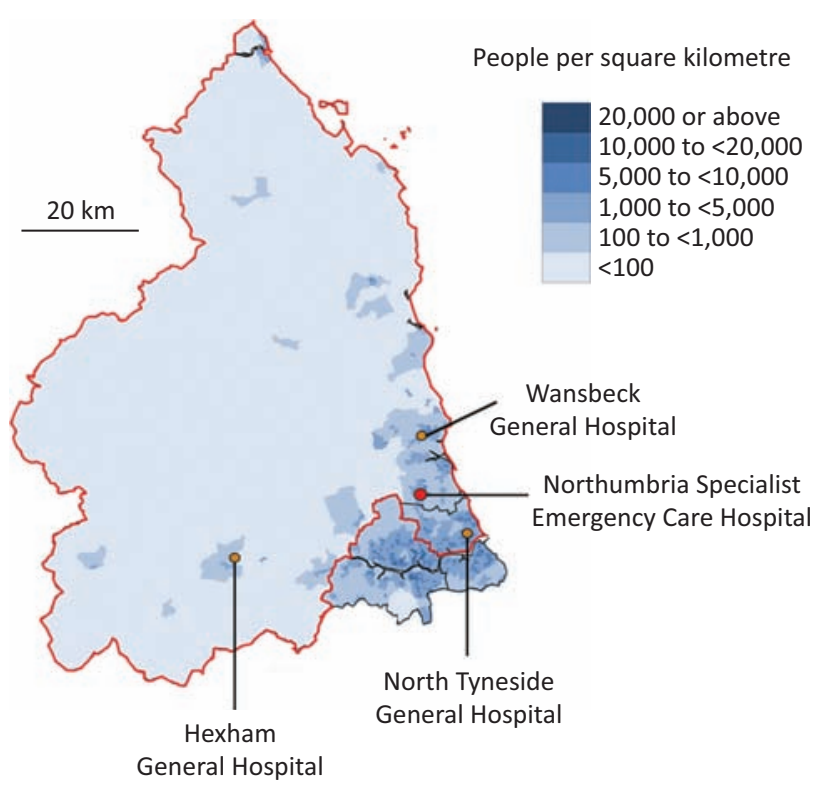

Fig 1. Map of population density across Northumberland and Tyne and Wear counties. The Northumbria Healthcare Trust boundary is highlighted in red. This image is modified from a figure published by the Office for National Statistics. ${ }^{13}$ It contains National Statistics data and Ordnance Survey data $\left({ }^{\odot}\right.$ Crown copyright and database right 2013). It also contains public-sector information licensed under the Open Government Licence v3.0.

Centralisation of acute stroke services has been shown to reduce mortality and length of stay, but results are not consistent across regional reorganisations. ${ }^{3,10,11}$ Data on the impact of centralisation upon dependency post stroke are limited. ${ }^{12}$

We conducted an interrupted time series analysis to establish whether the centralisation of multisite acute services into a single site hyperacute stroke service was associated with changes in key emergency care processes (scan time and thrombolysis delivery) and related outcomes (mortality, dependency and length of stay).

\section{Methods}

\section{Study setting}

Northumbria Healthcare NHS Foundation Trust (NHCT) provides secondary healthcare services to a rural and urban population of 500,000 people in North East England (Fig 1). ${ }^{13}$ Prior to June 2015 , 
emergency stroke care was provided in mixed acute stroke and rehabilitation units at three district general hospitals. All of these hospitals followed the same pre-hospital protocol involving a single NHS ambulance trust and admitted stroke patients to a combined acute and rehabilitation unit from their accident and emergency (A\&E) departments or medical admissions units. The patients then remained in the acute stroke and rehabilitation units throughout their inpatient stay. Each hospital had a site-based stroke consultant and stroke nurse practitioner available $9 \mathrm{am}-5 \mathrm{pm}$ Monday to Friday. An on-call stroke consultant visited each of the three sites daily at weekends and telemedicine was used for out-of-hours thrombolysis decisions. A consultant opinion regarding thrombolysis was available 24 hours a day in a cross-site rota, but this was often a remote review with thrombolysis being delivered by the A\&E staff.

In June 2015, the A\&E departments and all emergency services from the three acute hospitals were moved to a new purposebuilt hospital, Northumbria Specialist Emergency Care Hospital (NSECH), located in the south-east of the catchment area. NSECH provides consultant-led emergency care for the first 72 hours, and those who need ongoing inpatient care are then transferred to one of the original three local hospitals. Geographical modelling using the postcodes of previous emergency admissions informed by population density and traffic conditions located NSECH where there would be no overall net increase in travel times for the population served. There was no intentional change to the trust boundary or to pre-hospital ambulance provision.

NSECH has an 11-bed hyperacute stroke unit (HASU). A stroke consultant and nurse practitioner are available on site 12 hours a day, seven days a week, and a stroke consultant provides an overnight telemedicine service. There are dedicated emergency computed tomography (CT) and magnetic resonance imaging (MRI) scanners adjacent to the emergency department. Thrombectomy is available on an ad hoc basis at the regional neuroscience centre, which is located 10 miles away. A 24/7 thrombectomy service was not available during the study period and only a small number of patients (fewer than 30) were referred for consideration of thrombectomy. These patients are not included in the current dataset.

\section{Study design, participants and data collection}

We undertook an interrupted time series analysis covering 57 months (1 April 2013 to 31 December 2017). The data has been collected prospectively for the Sentinel Stroke National Audit Programme (SSNAP) database as part of routine clinical care and quality improvement for all confirmed stroke admissions. ${ }^{12}$ Patients with unknown stroke onset times or onset during sleep were excluded from the analysis of time from stroke onset to hospital arrival, but were included in all other analyses.

Discharge data were used to calculate mortality, dependency and total length of stay, the last being calculated using data from the last hospital in the trust that patients were discharged from (for example, if patients were transferred from NSECH to one of the district general hospitals, data from the district general hospitals were used).

Ethical approval for this study was not required as we used service data routinely collected quality improvement purposes by a single NHS trust. All data was provided in an anonymised format with approval from the trust information governance lead. Caldicott approval was obtained for use of data collected for the SSNAP.

\section{Statistical analyses}

Segmented regression models were used to establish the following outcomes: (i) time trends (change per month) for data prior to the centralisation (1 April 2013 to 30 June 2015); (ii) step change in levels for data immediately after centralisation (between 30 June 2015 and 1 July 2015); (iii) change in trends between data recorded before and after centralisation; and (iv) time trends (change per month) for data recorded after centralisation (1 July 2015 to 31 December 2017).

Dummy variables were included in the regression model to allow assessment of seasonal variation, and we considered the presence of positive or negative autocorrelation with the Durbin-Watson statistic (d), that is associations between consecutive monthly points in the time series data which indicate that error terms (residuals) in the regression models are not independent from one another and could confound results. In cases where $d>d_{u}$ or $4-d>$ $d_{u}$ (where $d_{u}$ is the upper critical value), it was concluded that there was no statistical evidence of positive or negative autocorrelation, respectively. $^{14}$

\section{Results}

A total of 4,305 acute stroke patients were admitted to Northumbria Healthcare NHS Foundation Trust between 1 April 2013 and 31 December 2017: 2,045 prior to the centralisation of acute stroke services and 2,260 post-centralisation. Among these patients, 528 (12.3\%) patients received treatment with intravenous thrombolysis during this period (Supplementary file 1). No evidence of seasonality or autocorrelation was found in any of the segmented regression models.

The trend for the number of patients admitted in the precentralisation period was increasing significantly by 0.61 patients per month ( $95 \%$ CI: 0.1 to 1.2). The number of admissions did not significantly change immediately after centralisation, and there was no significant trend for number of patients admitted over time in the post-centralisation period (Supplementary file 2). There were no statistically significant changes in the proportion of patients who received thrombolysis across the study periods.

Table 1 and Supplementary file 3 show the pre-centralisation trend, the step change at the centralisation point, the change in trends between data recorded before and after centralisation, and post-centralisation trends for inpatient mortality and dependency as assessed by modified Rankin Scale (mRS) at discharge for all acute stroke patients. ${ }^{15}$ There was no statistically significant impact of centralisation on mortality and dependency. Table 1 and Fig 2 demonstrate a statistically significant shortening of total length of stay immediately after centralisation of 4.9 days (95\% CI: -8.1 to -1.7 ), which was subsequently sustained over time during the post-centralisation period.

The time of stroke onset was known for 3,168 (73.6\%) patients (Fig $3 a$ ). The prolonged times between stroke onset and admission observed between 1 July and 30 November 2013 were due to an anomalous period of ambulance diversion. Interrupted time series analyses are particularly sensitive to outliers. ${ }^{16}$ The results of analyses with and without outlying months included are shown in Supplementary file 2. After excluding the outlying months, time from stroke onset to admission did not significantly change throughout the study periods.

Time from admission to brain imaging did not change across the whole cohort (Supplementary file 4), but those who received 
Table 1. Impact of centralisation upon mortality, dependency ${ }^{a}$ and length of stay

\section{$\%$ patients who died as an inpatient}

Pre-centralisation trend (units/

time)

Step change immediately after centralisation (units)

Change in trends (units/time)

Post-centralisation trend (units/ time)

$-0.2 \%$ /month (95\% CI: -0.3 to 0.0$)^{b}$

$0.5 \%$ (95\% CI: -3.3 to 4.3 )

$0.2 \%$ /month ( $95 \% \mathrm{CI}:-0.0$ to 0.4 )

to 0.3$)$
${ }^{a}$ Dependency as measured by the modified Rankin Scale ${ }^{15}$ (mRS): 0 - no symptoms; 1 - no significant disability despite symptoms, able to carry out all usual duties and activities; 2 - slight disability, unable to carry out all previous activities, but able to look after own affairs without assistance; 3 - moderate disability, requiring some help, but able to walk without assistance; 4 - moderately severe disability, unable to walk without assistance and unable to attend own bodily needs without assistance; 5 - severe disability, bedridden, incontinent and requiring constant nursing care; 6 - dead.

b $95 \%$ confidence intervals. Statistical significance was designated as $p<0.05$ where confidence intervals were borderline at one decimal place. thrombolysis had admission-to-scan times immediately after centralisation that were shortened by 16.2 minutes (95\% CI: -22.0 to -10.4$)$. This improvement was subsequently sustained over time during the post-centralisation period (Supplementary file 5).

Immediately after centralisation, there was a statistically significant (step change) improvement in time from admission to thrombolysis of 26.0 minutes (95\% CI: -39.9 to -12.1 ), which was subsequently sustained over time during the post-centralisation period (Supplementary file 4). However, this improvement in time trend during post-centralisation was non-significant when time from stroke onset to thrombolysis was analysed ( -21.3 minutes [95\% CI: -48.9 to 6.3]) (Fig 3b).

In Supplementary file 6, we have provided published SSNAP results comparing Northumbria Healthcare NHS Foundation Trust against the national averages for time from admission to scan and for time from admission to thrombolysis for the duration of the study. ${ }^{17}$ These data show that door-to-needle

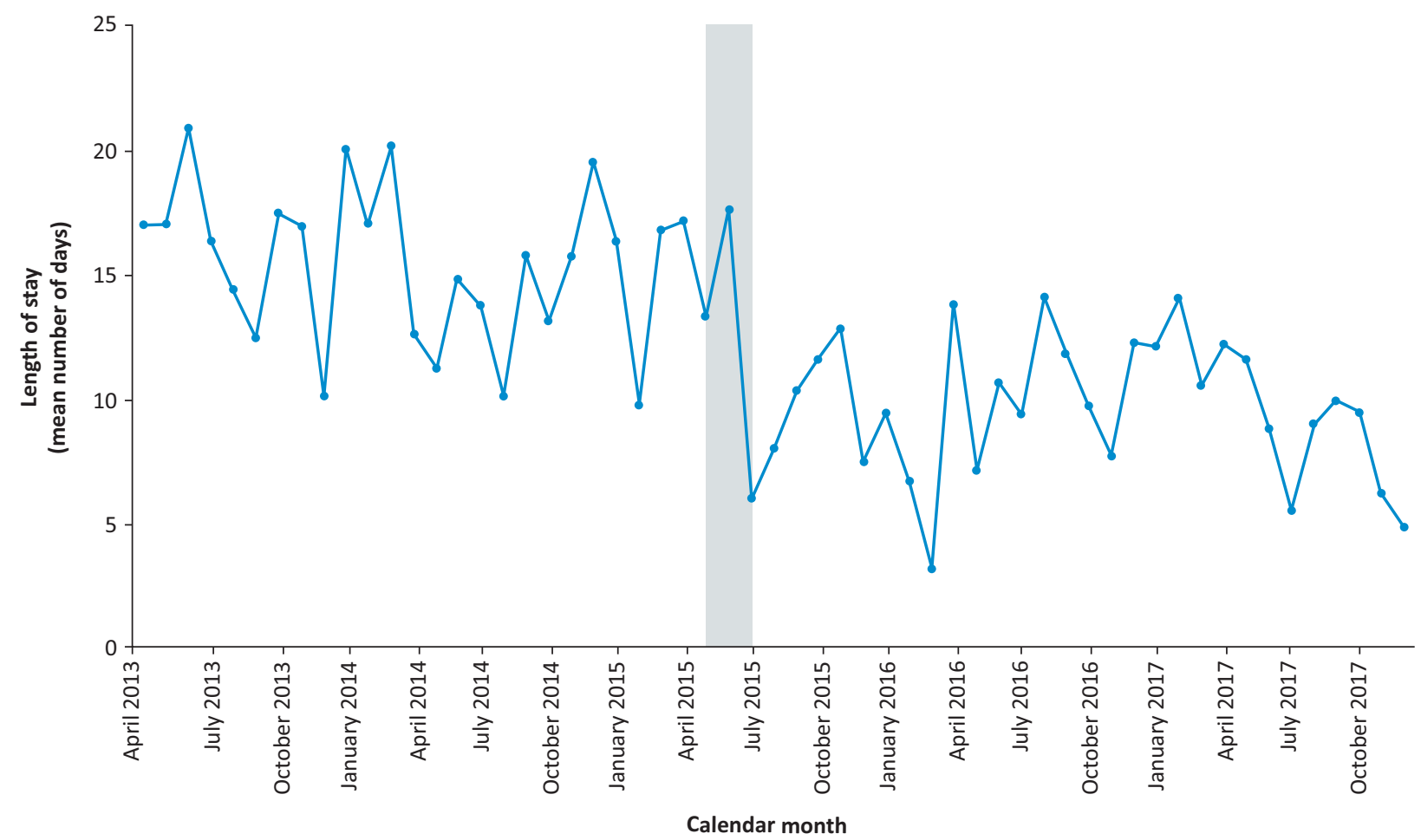

Fig 2. Total length of hospital stay from first admission to last discharge (mean days). The month during which centralisation took place (June 2015) is highlighted in grey. 

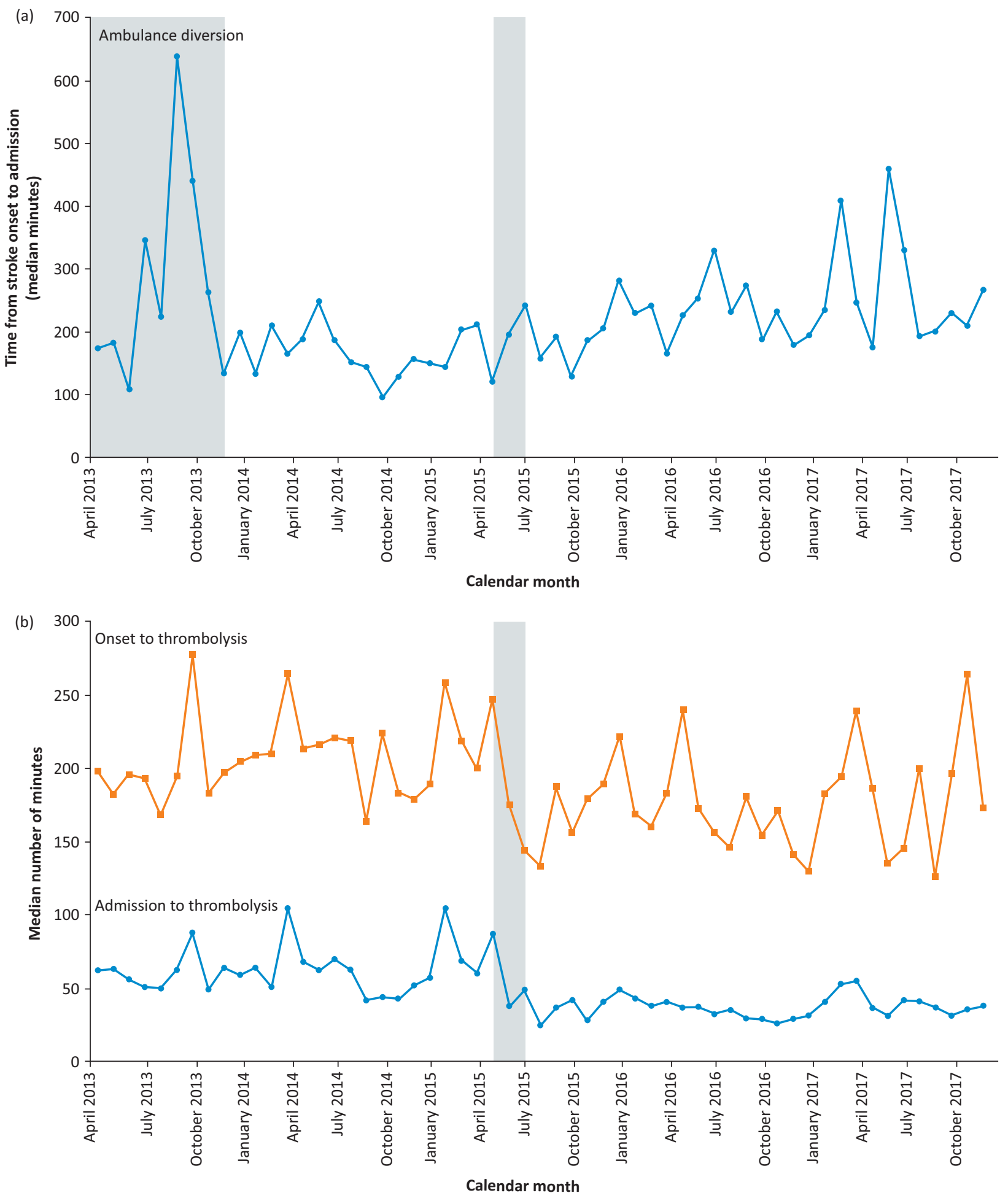

Fig 3. (a) Time from stroke onset to hospital admission (median minutes). A period of ambulance diversion due to CT scanner maintenance is highlighted in grey in the left part of the graph. Centralisation month (June 2015) is highlighted in grey. (b) Time from stroke onset to thrombolysis (top) and time from hospital admission to thrombolysis (bottom) are expressed as median minutes. Centralisation month (June 2015) is highlighted in grey.

times were generally longer than the national average prior to centralisation, but post-centralisation they have remained shorter than the national average. Case-mix data are available in Supplementary file 7.

\section{Discussion}

The regional centralisation of three acute stroke services in a large urban and rural NHS trust in North East England was associated 
with a statistically significant reduction in length of stay. Mortality and dependency outcomes were unchanged. Immediate improvements after centralisation were identified in door-toneedle times and in door-to-scan times for patients who received thrombolysis, and these improvements were sustained over time during the post-centralisation period.

The parameter estimates in the segmented regression models take into account the underlying time trends prior to centralisation. Therefore, the statistically significant changes in time trends after centralisation are over and above any background time trends. This increases the confidence that the estimates of effect that we identified can be attributed to the impact of centralisation.

A previous modelling exercise looking at acute stroke service reorganisation within North East England had shown a positive trade-off between longer ambulance journeys and improved thrombolysis delivery following theoretical redirection from eight local hospitals to two large HASUs. ${ }^{18}$ This analysis assumed that ambulance resources and geographical boundaries remained constant. Following the opening of Northumbria Specialist Emergency Care Hospital, there was no significant net change in the time between stroke onset and arrival at hospital. There may, however, have been variable effects for subgroups within the population due to changes in travel times to hospital from different localities and shifts in ambulance service provision to achieve response targets after centralisation. A more in-depth analysis of stroke onset-to-admission times should be explored in the future, which would require additional ambulance operational data and comparisons with other sites to consider the impact of chronological trends in service demand and performance.

Earlier scanning has also been shown to improve outcomes for acute stroke patients. ${ }^{19}$ Door-to-scan times were unchanged following centralisation when all patients were included in the analysis but were significantly reduced for the subset of patients who received thrombolysis, reflecting early coordinated clinical triage assessments. The observed statistically significant improvement in length of stay reflects the benefit obtained from focusing on forward care planning at an early stage, which was facilitated by twice-daily (including at weekends) multidisciplinary team discussions about potential discharges and good access to investigations, eg MRI and Doppler.

Intravenous thrombolysis is a time-dependent treatment and the sooner it is delivered the greater the benefit. ${ }^{20}$ Minimising transport delays, efficient paramedic and hospital assessments, and prompt delivery of imaging and thrombolysis itself are key to optimising the benefits of this treatment. ${ }^{21,22}$ Both before and after centralisation, thrombolysis was predominantly delivered by a consultant stroke physician and a stroke nurse practitioner. Increased on-site availability of these specialists probably contributed to further improvement in rapid thrombolysis assessments, to an extent over and above any improvement due to the greater experience that comes with higher volumes of patients being thrombolysed in a single centre. ${ }^{23}$ Acute radiology facilities (CT and MRI) were located next to the accident and emergency department, enabling quick access to scanning with pre-alert from paramedics. A streamlined care pathway contributed to the reduction in door-to-needle time.

\section{Methodological considerations}

An interrupted time series design enabled us to establish the effects of the centralisation of stroke services above and beyond any baseline time trends. ${ }^{16}$ An interrupted time series analysis ideally requires 10 or more pre-intervention time points. ${ }^{16}$ We included data for 27 months before and 30 months after centralisation. Other features of a robust analysis were: the inclusion of continuous variables, consideration of homoscedasticity, a lack of autocorrelation, and the normal distribution of residuals. ${ }^{24}$

Interrupted time series analyses are able to control for potential confounders provided that these confounders did not also change at the point of intervention. ${ }^{16}$ We have not identified any confounding factors that changed at the point of centralisation that may have impacted upon the results.

\section{Comparison with other studies}

Previous UK studies have reported before-and-after study data and difference-in-differences relating to the reorganisation of acute stroke services. ${ }^{3,10-12}$ This is the first report of an interrupted time series that addresses the potential methodological drawbacks of the alternative study designs. Our results are consistent with previous studies that have demonstrated that, when properly implemented, a centralised model of stroke care can achieve shorter length of stay and can improve key time metrics when compared to local service provision., 30-12

In London, stroke care for a population of 8.17 million people was reorganised into eight HASUs for all suspected stroke admissions. After a period of up to 72 hours, patients who required further stroke specialist care were transferred to one of 24 local stroke units. ${ }^{25}$ This major reorganisation led to an improvement in 90-day mortality of $1.1 \%$ (95\% CI: -2.1 to -0.1 ) above that seen in the rest of England, including reductions in hospital stay by a mean of 1.4 days ( $95 \%$ CI: -2.3 to 0.5 ), which contributed to significant cost savings. 3,10

The Greater Manchester (population 2.6 million) reorganisation was less radical in that services were only centralised for patients presenting within the time window for potential thrombolysis. Patients presenting less than 4 hours after a suspected stroke were taken to one of two primary stroke centres (offering thrombolysis for 12 hours a day, 5 days a week) or alternatively to one comprehensive stroke centre (offering hyperacute stroke and regional neuroscience services 24 hours a day, 7 days a week). Patients were then discharged or transferred to their local stroke unit within 72 hours. Patients presenting more than 4 hours after a suspected stroke were taken to one of 10 local district stroke centres (DSC). ${ }^{25}$ There was no improvement in mortality but length of stay was reduced by a mean of 2.0 days ( $95 \%$ CI: -2.8 to -1.2 ) as a result of this reorganisation. ${ }^{10}$

The context of our study is positioned between a very local and a regional setting as we report the reorganisation of three sites within a single large NHS trust without crossing organisational boundaries. Unlike the stroke service reconfigurations that have been assessed previously, this centralisation was part of the comprehensive restructuring of all of the acute services within the region: Northumbria Specialist Emergency Care Hospital is the first purpose-built Emergency Care Hospital in England. ${ }^{26}$ Our findings are consistent with the results from London and Greater Manchester, which also found that centralisation improved length of hospital stay. We are also able to provide evidence that centralisation improved door-to-needle times for patients who received thrombolysis. We did not find any statistically significant difference in mortality or dependency following centralisation. 


\section{Strengths and limitations of this study}

A major strength of our study was that stroke care was provided by the same stroke consultants and nurse practitioners before and after centralisation. We also maintained consistency of measurements across the whole study period with a standardised approach to data collection being used by the same healthcare professional workforce across the study period. The SSNAP cross-references locally submitted cases via the Office of National Statistics to ensure high levels of ascertainment for stroke admissions and openly reports data completeness. ${ }^{12}$

We chose to undertake an interrupted time series study because this design provides an efficient but strong methodology that employs readily available data. ${ }^{27}$ A randomised controlled trial would be the gold standard to assess the impact of the centralisation of stroke services upon outcomes of care. However, a multicentre trial to evaluate the centralisation of stroke services would be significantly more expensive, would be logistically challenging, would take longer to perform and would carry its own ethical dilemmas. ${ }^{28}$

We used health status at discharge from hospital as a key outcome measure. Including longer-term outcomes could have strengthened the study but these data were not available. In addition, a single group interrupted time series analysis cannot correct for any confounding factors that also changed at the centralisation point, whereas a comparative interrupted time series analysis can achieve this correction. ${ }^{29}$

Our focus was on aspects of service delivery that could be particularly influenced by a change in emergency care processes within the first hour of hospital admission, but a larger study could consider the impact of other acute care processes such as swallowing assessment and provision of fluid support.

\section{Implications}

Acute stroke care is one of five specialist services priorities for urgent and emergency care networks in England. ${ }^{30}$ Acute stroke services are delivered across 155 sites $^{31}$ in England and many are struggling to provide $24 / 7$ stroke specialist care. Services with higher rates of stroke admissions have previously been shown to be both more efficient and more effective. ${ }^{23}$ The centralisation of services is a controversial topic for patients, communities and politicians ${ }^{32}$ but this study provides further robust research evidence to support the case for change.

\section{Conclusions}

We found evidence that the centralisation of three acute stroke units within a single NHS trust in North East England improved door-to-needle times and reduced the average length of inpatient stay. This supports the ongoing re-organisation of emergency care for stroke patients as an NHS priority.

\section{Funding}

This study was funded by Newcastle University and Health Education North East. CP receives a fellowship funded by the Stroke Association.

\section{Author contributions}

All authors were involved in the conception and design of the work. CP provided the data which were analysed by ME with help from DF. All authors were involved in interpreting the results and contributed to the drafting, critical revision and final approval of the article.

\section{Conflicts of interest}

$\mathrm{CP}$ and $\mathrm{HR}$ are consultant stroke physicians at Northumbria Health Care NHS Foundation Trust.

\section{Data sharing}

All authors had full access to all of the data in the study and can take responsibility for the integrity of the data and the accuracy of the data analysis. No additional data are available.

\section{Supplementary material}

Additional supplementary material may be found in the online version of this article at http://futurehospital.rcpjournal.org:

S1 - Graph of numbers of patients admitted (top) and thrombolysed (bottom). Centralisation month (June 2015) is highlighted in grey.

S2 - Table of number of stroke admissions, proportion thrombolysed, and impact of centralisation upon time from stroke onset to admission.

S3 - Graphs of inpatient mortality (top) and dependency at discharge (bottom). (a) Inpatient mortality expressed as a percentage of all acute stroke admissions. Centralisation month (June 2015) is highlighted in grey. (b) Dependency at discharge for all acute stroke admissions expressed on a modified Rankin Scale. Centralisation month (June 2015) is highlighted in grey.

S4 - Table of impact of centralisation upon door-to-scan and thrombolysis times.

S5 - Graph of time from admission to brain imaging. Time from hospital admission to CT head scan for all patients (top) and for thrombolysed patients (bottom) expressed as median minutes. Centralisation month (June 2015) is highlighted in grey.

S6 - Table of quarterly door-to-needle and door-to-scan times from published SSNAP data.

S7 - Table of casemix pre- and post-centralisation.

\section{Acknowledgements}

The authors would like to acknowledge the members of the stroke service at Northumbria Health Care NHS Foundation Trust, Sentinel Stroke National Audit Programme and the Office for National Statistics. Professor Helen Rodgers will act as guarantor for the paper.

\section{References}

1 Stroke Unit Trialists' Collaboration. Organised inpatient (stroke unit) care for stroke. Cochrane Database Syst Rev 2013;9:CD000197.

2 Price CI, Clement F, Gray J, Donaldson C, Ford GA. Systematic review of stroke thrombolysis service configuration. Expert Rev Neurother 2009;9:211-33.

3 Hunter RM, Davie C, Rudd A et al. Impact on clinical and cost outcomes of a centralized approach to acute stroke care in London: a comparative effectiveness before and after model. PLoS One 2013;8:e70420.

4 Norrving B, Adams RJ. Organized stroke care. Stroke 2006;37:326-8.

5 Alberts MJ, Hademenos G, Latchaw RE et al. Recommendations for the establishment of primary stroke centers. Brain Attack Coalition. JAMA 2000;283:3102-9.

6 Alberts MJ, Latchaw RE, Selman WR et al. Recommendations for comprehensive stroke centers: a consensus statement from the Brain Attack Coalition. Stroke 2005;36:1597-616.

7 Kelly C, Hulme C, Farragher T, Clarke G. Are differences in travel time or distance to healthcare for adults in global north countries associated with an impact on health outcomes? A systematic review. BMJ Open 2016;6:e013059. 
8 Saver JL. Time is brain-quantified. Stroke 2006:37:263-6.

9 NHS England. Five year forward view. 2014. www.england. nhs.uk/wp-content/uploads/2014/10/5yfv-web.pdf [Accessed 29 September 2017].

10 Morris S, Hunter RM, Ramsay AI et al. Impact of centralising acute stroke services in English metropolitan areas on mortality and length of hospital stay: difference-in-differences analysis. BMJ 2014:349:g4757.

11 Ramsay AI, Morris S, Hoffman A et al. Effects of centralizing acute stroke services on stroke care provision in two large metropolitan areas in England. Stroke 2015;46:2244-51.

12 Sentinel Stroke National Audit Programme. Rising to the challenge: the fourth SSNAP annual report. SSNAP, 2017.

13 Office for National Statistics. Super output area mid-year population estimates, mid-2012. ONS, 2013. www.ons.gov.uk/ons/ dcp171778_330327.pdf [Accessed 29 September 2017].

14 Savin NE, White KJ. The Durbin-Watson test for serial correlation with extreme sample sizes or many regressors. Econometrica 1977:45:1989-96.

15 Banks JL, Marotta CA. Outcomes validity and reliability of the modified Rankin scale: implications for stroke clinical trials: a literature review and synthesis. Stroke 2007;38:1091-6.

16 Wagner AK, Soumerai SB, Zhang F, Ross-Degnan D. Segmented regression analysis of interrupted time series studies in medication use research. J Clin Pharm Ther 2002;27:299-309.

17 Sentinel Stroke National Audit Programme. Clinical Audit Results. www.strokeaudit.org/results/Clinical-audit.aspx [Accessed 23 February 2018].

18 McMeekin P, Gray J, Ford GA, Rodgers H, Price CI. Modelling the efficiency of local versus central provision of intravenous thrombolysis after acute ischemic stroke. Stroke 2013:44:3114-9.

19 Wardlaw JM, Seymour J, Cairns ] et al. Sandercock P. Immediate computed tomography scanning of acute stroke is cost-effective and improves quality of life. Stroke 2004;35:2477-83.

20 Saver JL, Smith EE, Fonarow GC et al. The golden hour and acute brain ischemia: presenting features and lytic therapy in over 30,000 patients arriving within 60 minutes of onset. Stroke 2010;41:1431-9.

21 Meretoja A, Kaste M. Pre- and in-hospital intersection of stroke care. Ann N Y Acad Sci 2012;1268:145-51.

22 Meretoja A, Keshtkaran M, Saver JL et al. Stroke thrombolysis: save a minute, save a day. Stroke 2014:45:1053-8.
23 Bray BD, Campbell J, Cloud GC et al. Bigger, faster? Associations between hospital thrombolysis volume and speed of thrombolysis administration in acute ischemic stroke. Stroke 2013:44:3129-35.

24 Laerd Statistics. Simple Linear Regression in SPSS Statistics. 2013. https://statistics.laerd.com/spss-tutorials/linear-regression-usingspss-statistics.php [Accessed 3 August 2016].

25 Fulop NJ, Ramsay AI, Perry C et al. Explaining outcomes in major system change: a qualitative study of implementing centralised acute stroke services in two large metropolitan regions in England. Implement Sci 2016;11:80.

26 Northumbria Health Care Trust. A new era in emergency care. 2015. www.northumbria.nhs.uk/emergency/ [Accessed 17 ]uly 2017].

27 Kreis I, Busby A, Leonardi G, Meara J, Murray V. Essentials of environmental epidemiology for health protection: a handbook for field professionals. Oxford: Oxford University Press, 2012.

28 Weijer C, Grimshaw JM, Taljaard M et al. Ethical issues posed by cluster randomized trials in health research. Trials 2011;12:100.

29 Lopez Bernal J, Cummins S, Gasparrini A. Interrupted time series regression for the evaluation of public health interventions: $a$ tutorial. Int J Epidemiol 2016:46:348-55.

30 NHS England. Role and establishment of urgent and emergency care networks. 2015. www.nhs.uk/NHSEngland/keogh-review/ Documents/Role-Networks-advice-RDs \% 201.1FV.pdf [Accessed 28 September 2017].

31 Sentinel Stroke National Audit Programme (SSNAP). Acute organisational audit report, November 2016. www.strokeaudit.org/ Documents/National/AcuteOrg/2016/2016-AOANationalReport. aspx [Accessed 28 September 2017].

32 Bouverie J. Major changes in stroke care can save lives. 2017. www. england.nhs.uk/blog/major-changes-in-stroke-care-can-save-lives/ [Accessed 29 September 2017].

Address for correspondence: Professor Helen Rodgers, Stroke Research Group, Institute of Neuroscience, Newcastle University, 3-4 Claremont Terrace, Newcastle-upon-Tyne NE1 7RU, UK.

Email: helen.rodgers@newcastle.ac.uk 